\title{
Spontaneous perforation of common bile duct in infants
}

\author{
E. R. HOWARD, D. I. JOHNSTON, and A. P. MOWAT \\ From the Departments of Surgery and Child Health, King's College Hospital Medical School, London
}

\begin{abstract}
Howard, E. R., Johnston, D. I., and Mowat, A. P. (1976). Archives of Disease in Childhood, 51, 883. Spontaneous perforation of common bile duct in infants. Two infants with spontaneous perforation of the common bile duct are described. One presented with mild jaundice, dark urine, acholic stools, and hydroceles, the other with bilateral inguinal hernia. In both the diagnosis was unsuspected until bile-stained ascites was discovered. Both eventually developed bile-staining of the scrotum. Neither was acutely ill. The 131I-Rose Bengal faecal excretion test showed reduced faecal excretion at $8 \%$ and $12 \%$ of the injected dose with 16.5 and $17 \% / \mathrm{dl}$ of the dose being recovered in the ascitic fluid 48 hours after intravenous injection. The ascitic:plasma ratio of isotope at that time was 32:1 and 28:1. Operative cholangiography in both showed a perforation at the junction of the cystic duct and common bile duct with no contrast entering the duodenum. Cholecystenterostomy using a Roux-en-Y loop of jejunum produced a rapid sustained recovery and is suggested as the treatment of choice. This condition should be considered in the differential diagnosis of obstructive jaundice in infancy since early surgical correction is necessary.
\end{abstract}

Although more than 50 cases of spontaneous perforation of the extrahepatic bile ducts in infants have been described (Lilly, Weintraub, and Altman, 1974), the disorder is infrequently listed as a cause of obstructive jaundice (deLorimier, 1973; SassKortsak, 1974) and thus may not be recognized though immediate surgical treatment is indicated. An awareness of the characteristic clinical picture, however, should allow prompt diagnosis and early treatment. Patients usually present in the first 3 months of life with mild jaundice and pale or acholic stools, or with abdominal distension and hernia, in addition to failure to thrive, irritability, and vomiting. Biochemical investigations show a conjugated hyperbilirubinaemia but either normal or only slightly abnormal hepatic transaminases. Surgical management is controversial, ranging from simple drainage to biliary diversion procedures. This communication describes 2 further cases emphasizing the typical clinical features, and discusses the use of ${ }^{131}$ I-Rose Bengal to establish the presence of a biliary leak. Prompt sustained recovery occurred after cholecystojejunostomy.

Case reports

Case 1. A male infant was referred at the age of 5

Received 1 March 1976. weeks for investigation of persistent jaundice. Pregnancy and delivery had been normal, birthweight $3.7 \mathrm{~kg}$, and his progress was normal until day 12 when he began to vomit and feed poorly. Jaundice developed on day 14, associated with pale stools, dark urine, and poor weight gain. When first examined he was jaundiced with a prominent umbilical hernia and bilateral scrotal hydroceles, but there was no demonstrable hepatosplenomegaly and he was apyrexial. Investigations included $\mathrm{Hb} 12 \cdot 1 \mathrm{~g} / \mathrm{dl}$, white blood count $183000 /$ $\mathrm{mm}^{3}\left(18.3 \times 10^{9} / \mathrm{l}\right)$ with $30 \%$ neutrophils, $62 \% \mathrm{lym}-$ phocytes, 5\% eosinophils, $2 \%$ monocytes. Total bilirubin was $156 \mu \mathrm{mol} / 1(9.1 \mathrm{mg} / 100 \mathrm{ml})$ with a conjugated bilirubin of $108 \mu \mathrm{mol} / 1(6.3 \mathrm{mg} / 100 \mathrm{ml})$. Alkaline phosphatase $350 \mathrm{IU} / 1$; aspartate amino transferase $183 \mathrm{IU} / 1$; prothrombin time $15 \mathrm{~s}$, control $15 \mathrm{~s}$; sweat sodium $17 \mathrm{mmol} / 1(17 \mathrm{mEq} / \mathrm{l})$. Barium meal and liver scan were normal. Percutaneous liver biopsy was abandoned when bile-stained ascites was aspirated during the introduction of local anaesthesia.

The infant was at this time in a stable clinical condition and it was decided to delay surgery until the completion of a ${ }^{131}$ I-Rose Bengal excretion study. After intravenous administration of $5 \mu \mathrm{Ci}$ of the isotope, the 72-hour stool content was $8 \cdot 4 \%$ (within the range of total obstruction to bile flow). At 48 hours the ascitic fluid activity was equivalent to $16.5 \%$ of the administered isotope per $\mathrm{dl}$ and the ratio of ascitic fluid to plasma activity was $32: 1$. During this period the umbilicus and the hydroceles became strikingly bile-stained (Fig. 1). 


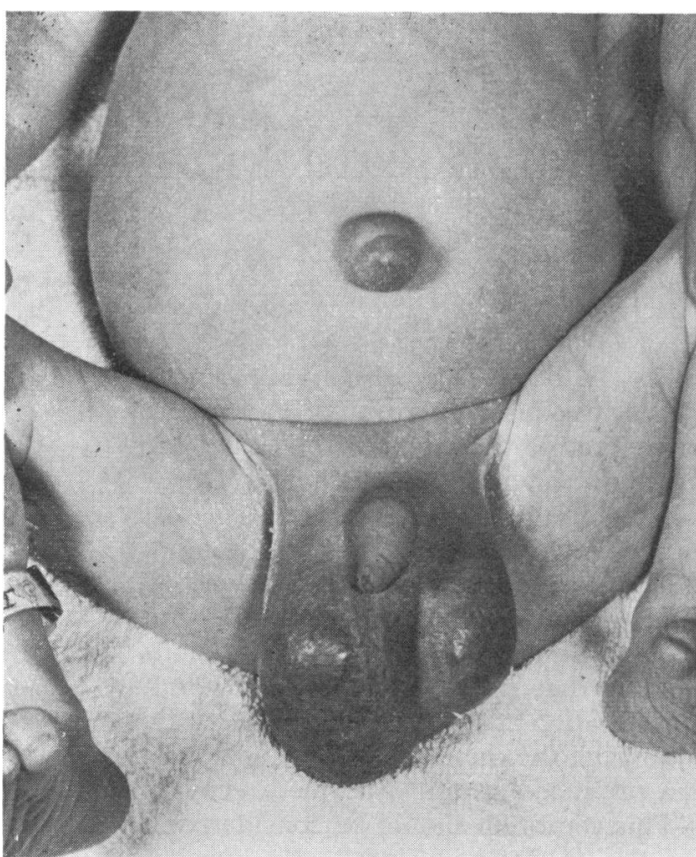

FIG. 1.-Case 1. Bile-stained hydroceles and umbilical hernia in a 5-week-old infant with abdominal distension.

At laparotomy there was clear bile-stained ascites and a large bile-containing cyst of the lesser sac. The gallbladder was distended with bile. An operative cholangiogram via the gallbladder showed a normal pattern in the intrahepatic biliary tree but no flow from the distal common bile duct into the duodenum. Contrast medium leaked from a perforation at the junction of the cystic duct with the common bile duct and entered a cavity in the lesser sac (Fig. 2). No further exploration of the distal common bile duct was made. The gallbladder was anastomosed to a Roux-en-Y loop of jejunum and the lesser sac and subhepatic spaces were drained. Histological examination of a surgical liver biopsy showed a normal lobular pattern but marked cholestasis. The portal tracts were oedematous and there was some bile duct proliferation. These features were consistent with a diagnosis of large duct obstruction.

The postoperative period was uneventful and oral feeding was restarted on the fourth day. A mediumchain triglyceride milk formula (Portagen, Mead Johnson) was prescribed together with cholestyramine and phenobarbitone. The jaundice resolved rapidly and the ascites did not recur. At one year of age he is in excellent health, on a mixed diet, with normal liver function tests.

Case 2. A 5-week-old male infant was found to have bile-stained ascites at the time of a bilateral inguinal herniotomy. Pregnancy and delivery had been normal,

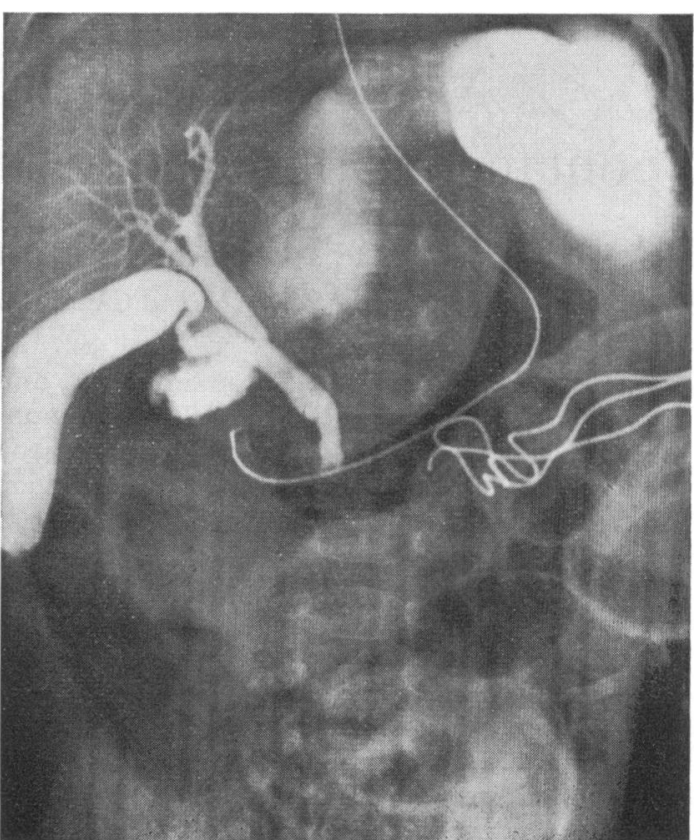

FIG. 2.-Case 1. Operative cholangiogram showing a perforation of the common bile duct at the junction with the cystic duct. The contrast medium flows freely into the lesser sac and accumulates in the splenic area, but there is no flow into the duodenum. Nasogastric tube and sponge markers are present.

birthweight $3.4 \mathrm{~kg}$, but abdominal distension, inguinal swellings, and repeated vomiting had occurred since the first week. Stools were pale but jaundice was not observed, there was no hepatosplenomegaly, and ascites was not recognized clinically. Investigations included $\mathrm{Hb} 8.4 \mathrm{~g} / \mathrm{dl}$, WBC $14400 / \mathrm{mm}^{3}\left(14.4 \times 10^{9} / \mathrm{l}\right)$ with $68 \%$ neutrophils, $29 \%$ lymphocytes, $2 \%$ eosinophils, and $1 \%$ monocytes. Total bilirubin $117 \mu \mathrm{mol} / \mathrm{l}$ $(6.8 \mathrm{mg} / 100 \mathrm{ml})$, direct bilirubin $79 \mu \mathrm{mol} / 1(4.6 \mathrm{mg} / 100$ $\mathrm{ml})$; alkaline phosphatase $457 \mathrm{IU} / 1$; aspartate transferase $30 \mathrm{IU} / 1$; prothrombin time $18 \mathrm{~s}$, control $15 \mathrm{~s}$. Barium meal suggested anterior displacement of the duodenum but no abnormal masses were defined on liver scan. ${ }^{131}$ I-Rose Bengal excretion study using $5 \mu \mathrm{Ci}$ of isotope resulted in a 72-hour stool activity of $12.8 \%$ (just above the total obstruction range) and an ascitic fluid activity of $17 \cdot 2 \%$ of the administered dose per $\mathrm{dl}$ at 24 hours. The corresponding ascites : plasma activity ratio was $28: 1$.

At laparotomy there were more than $150 \mathrm{ml}$ of clear bile-stained ascites. The gallbladder wall was thickened and the hepatic ducts were dilated. Cholangiography showed a leak in the common bile duct at its junction with the cystic duct and free flow of the dye into the peritoneal cavity. There was no flow from the distal bile duct into the duodenum. The surgical technique was similar to that in Case 1 with the creation of a 
cholecystojejunostomy and peritoneal drainage. Needle biopsy of the liver showed widening of the portal tracts with deposition of fibrous tissue. The infant had an uneventful recovery from surgery and is well on a normal diet 7 months later. Liver function tests are normal.

\section{Discussion}

Spontaneous perforation of the common bile duct and bile peritonitis in early infancy is a well documented entity but its relative rarity and the often insidious onset continue to make it a diagnostic problem. Of approximately 200 children studied as part of a prospective regional survey of infants with persistent conjugated hyperbilirubinaemia (Cottrall, Cook, and Mowat, 1974), only 2 presented with bile peritonitis. Though the clinical features of these infants resembled those described by other authors (Johnston, 1961; Lees and Mitchell, 1966), diagnosis was delayed in both cases.

The birth and neonatal periods are generally unremarkable and are followed by a variable interval of apparently normal health. Infants usually present between 1 week and 2 months but some cases have been reported as late as 30 months (Hindmarsh, 1947; Lees and Mitchell, 1966). Initial signs may include mild fluctuating jaundice accompanied by pale stools and dark urine but this is not consistent, and there may be a nonspecific picture of poor weight gain, repeated vomiting, and irritability. Progressive abdominal distension, especially when accompanied by bile-staining of hydroceles, inguinal herniae, or abdominal wall is a characteristic feature. The general clinical condition is usually good and only a minority of cases have pyrexia or significant leucocytosis. Diagnostic paracentesis will usually show clear bile-stained ascites but the staining may not be obvious early in the disease (Wynne et al., 1968).

The relatively low bilirubin in the presence of acholic stools is an indication for correct diagnosis. Hepatic transaminases may be normal. 131I-Rose Bengal faecal excretion studies using a dose of $1 \mu \mathrm{Ci} / \mathrm{kg}$, with the measurement of the isotope ratio in ascitic fluid to serum is particularly helpful in diagnosis. This method is preferable to the scintiscanning technique in which the isotope, 48 hours after injection, is shown to be diffusely spread throughout the peritoneal cavity rather than confined to the descending colon (Hansen et al., 1974).

Controversy exists as to the optimum form of surgical management reflecting lack of understanding of the cause of spontaneous perforation. Viral infections (Moore, 1975) and fat necrosis from pancreatic reflux (Johnston, 1961) have been suggested as possible causes. The site of perforation is, however, very constant and Johnston (1961) postulated a developmental weakness at the junction of cystic and common bile ducts. Operative cholangiography frequently shows an obstruction at the lower end of the common bile duct which may be due to stenosis or segmental atresia, but in other cases such obstruction proves temporary and is usually attributed to inspissated bile or gallstones. In some patients, however, there is no apparent obstruction at laparotomy and a simple drainage procedure, with or without suture of the perforation, is followed by rapid improvement. The difficulty arises in the remaining patients who, if they have a permanent obstruction, require a cholecystenterostomy but if the obstruction is temporary may eventually establish normal bile drainage into the duodenum, as occurred in 15 of 53 cases reported in a recent review by Lilly et al. (1974). These authors suggested that simple drainage is the treatment of choice in all cases. The problems of this form of therapy were well illustrated by the 2 cases they reported. One died after two exploratory operations having had no spontaneous closure of the perforation, while the second case required two drainage procedures, had a persistent biliary fistula for 25 days, and required prolonged intravenous nutrition.

Ascending cholangitis is a risk after cholecystenterostomy and this operation should be avoided if possible, but the incorporation of a Roux-en-Y loop of jejunum in the procedure considerably reduces the risks of infection. If spontaneous resolution of the bile obstruction occurs the cholecystenterostomy is likely to close spontaneously. Cholecystenterostomy does have the major advantage that malabsorption due to lack of bile is immediately corrected and prolonged biliary drainage with the risk of bacterial contamination of the ascitic fluid is avoided.

Although spontaneous perforation of the bile ducts is a rare cause of obstructive jaundice in infancy it must be considered in all cases since death from bacterial contamination of the ascites is an ever present risk. In 2 patients with obstruction of the common bile duct a cholescystenterostomy using a Roux-en-Y loop of jejunum was followed by rapid and sustained postoperative recovery. We suggest that this mode of therapy rather than simple drainage is the treatment of choice in such patients.

\section{REFERENCES}

Cottrall, K., Cook, P. J. L., and Mowat, A. P. (1974). Neonatal hepatitis syndrome and alpha-1-antitrypsin deficiency: an epidemiological study in South-East England. Postgraduate Medical fournal, , 50, 376. 
deLorimier, A. A. (1973), Surgical causes of neonatal obstructive jaundice. New England fournal of Medicine, 288, 1284.

Hansen, R. C., Wasnich, R. D., De Vries, P., and Sunshine, P. (1974). Bile ascites in infancy: diagnosis with 131I-RoseBengal. Fournal of Pediatrics, 84, 719

Hindmarsh, F. D., (1947). Bile peritonitis in infancy. British Medical fournal, 2, 131.

Johnston, J. H. (1961). Spontaneous perforation of the common bile-ducts in infancy. British fournal of Surgery, 48, 532.

Lees, W., and Mitchell, J. E. (1966). Bile peritonitis in infancy. Archives of Disease in Childhood, 41, 188.

Lilly, J. R., Weintraub, W. H., and Altman, R. P. (1974). Spontaneous perforation of the extrahepatic bile ducts and bile peritonitis in infancy. Surgery, 75, 664.
Moore, T. C. (1975). Massive bile peritonitis in infancy due to spontaneous bile duct perforation, with portal vein occlusion. fournal of Pediatric Surgery, 10, 537.

Sass-Kortsak, A., (1974). Management of young infants presenting with direct-reacting hyperbilirubinemia. Pediatric Clinics of North America, 21, 777.

Wynne, J. M., Cywes, S., Retief P. J. M., and Louw, J. H. (1968). Ascites in the newborn. South African Medical fournal, 42, 919.

Correspondence to Dr. Alex P. Mowat, Department of Child Health, King's College Hospital Medical School, Denmark Hill, London SE5 8RX. 